\title{
Autoregulated Combined Resistance Training for Plantar Heel Pain in Athletes: Protocol for a Feasibility Prospective Cohort Study
}

Ian Burton MSc, CSCS

Physiotherapist, Angus HSCP, NHS Tayside, Dundee, United Kingdom

Please cite as: Burton, I. (2020). Autoregulated Combined Resistance Training for Plantar Heel Pain in Athletes: Protocol for a Feasibility Prospective Cohort Study. SportRxiv doi: 10.31236/osf.io/q87xe

\begin{abstract}
Background: Musculoskeletal disorders such as tendinopathy are having an increasing burden on society and health systems. Plantar heel pain (PHP) is considered a tendinopathy and it affects up to $10 \%$ of the population, having a high incidence in running athletes. Although resistance training has shown shortterm effectiveness for treating PHP, more comprehensive exercise protocols and progression methods are needed due to poor long-term outcomes. Combining heavy slow resistance training with other types of exercise, such as plyometric exercise could lead to superior outcomes. It is recommended that PHP not be
\end{abstract}


treated by one exercise type in isolation. However, studies often use only one type of exercise, with there being a need to investigate feasibility and effectiveness of comprehensive exercise programs in PHP. Resistance training programs in PHP are currently not achieving required intensity and dosage, leading to high recurrence rates. Therefore, better methods for progressing resistance training are required to improve outcomes. One potential method is autoregulation, which allows individuals to progress at their own rate, taking individual factors into account. Despite being found effective in increasing strength in healthy athletes, autoregulation methods have not been investigated in tendinopathy.

Methods: A feasibility cohort study investigating a comprehensive exercise program for PHP could test feasibility and acceptability, prior to conducting a larger-scale study. A feasibility study would also help to determine if specific autoregulation progression methods for resistance training is feasible and acceptable for use with PHP patients. Patients expectations, and views on feasibility and acceptability of different types of exercise as well as autoregulation progression methods for PHP are unknown. Therefore, the addition of qualitative interviews in a mixed methods format would help ascertain acceptability of these methods and exercise interventions for the first time and help explain the intervention outcomes, allowing for design of a larger-scale study to determine effectiveness.

Keywords: Extracorporeal Shockwave Therapy: Resistance training; Tendinopathy; Fasciitis, Plantar.

\section{Background}

The global burden of pain and disability to individuals and costs to healthcare systems caused by musculoskeletal disorders is becoming increasingly more evident as they collectively comprise $21.3 \%$ of global volume of years lived with disability [1, 2]. In the United Kingdom (UK), musculoskeletal disorders are responsible for $25 \%$ of all general practitioner consultations, and $8 \%$ of total National Health Service (NHS) expenditure $[3,4]$. Foot related pain is one of the largest contributors to the musculoskeletal disease burden, with plantar heel pain (PHP) being the most prevalent foot-related musculoskeletal disorder, occurring 
in up to $10 \%$ of the general population $[5,6]$. The disorder was previously believed to be caused by an acute inflammatory response and termed plantar 'fasciitis', however chronic degeneration of fascia tissue due to repetitive microtrauma as seen in tendinopathy is considered a more likely pathophysiology, which leads to chronic pain and functional limitations [7-11]. Foot injuries are common among athletes, with the incidence of PHP in running athletes ranging from 4.5 to $10 \%$, and it accounts for $5 \%$ of injuries in novice runners and $11 \%$ in ultra-marathon running athletes $[12,13]$. The biomechanics of running, which involve vertical ground reaction forces acting on the foot can triple an athlete's bodyweight, with the plantar fascia and longitudinal arch involved in the force absorption mechanism [14-18]. There are no consensus recommendations for treating PHP in athletes, due to a dearth of studies conducted in this population, with specific studies necessary to determine optimal treatment strategies in athletes [19].

There are currently a range of passive treatment options for PHP used in clinical practice including, taping, manual therapy, orthotics, corticosteroid injections, platelet rich plasma or blood-derived injections, and electrotherapy approaches such as laser and shockwave therapy [20-30]. Exercise therapy targeting the injured tissue such as plantar fascia specific stretching (PFSS) and heavy slow resistance training (HSRT) have been found to be safe, clinically and cost-effective treatment options [30-35]. However, despite short-term effectiveness of exercise approaches, more effective interventions are warranted, to improve long-term outcomes which are currently inadequate $[36,37]$. Poor long-term outcomes may be due to patients not achieving the desired loading required to stimulate strength adaptations, suggesting more effective training methods are required [38].

A recent PHP study found that a self-dosed HSRT group did not increase the achieved exercise dose compared with a pre-determined protocol, with no differences in outcomes between the groups [38]. The authors concluded that isolated HSRT regimens are not sufficient to achieve satisfactory outcomes in PHP. Isolated HSRT does not address deficits in an individual's entire strength spectrum, with the addition of other exercise types such as plyometric and kinetic chain exercise being needed to address strength deficits [39]. Rehabilitation 
programs focusing solely on heel-raise protocols, may lead to suboptimal functional capabilities, persistent strength deficits, inadequate rehabilitation and ultimately high recurrence rates of tendinopathy [40-42]. Plyometric activities and exercise have been shown to be feasible and beneficial for improving outcomes in Achilles tendinopathy, but have not been investigated for PHP [43]. Kinetic chain strengthening targeting the gluteal muscles has also been found to be an effective exercise approach for PHP, however it is often not combined with HSRT heel-raise protocols [44]. Current literature recommends that combined rather than single interventions should be recommended for treating PHP to achieve good outcomes and patient satisfaction [45-48]. However, there is a dearth of studies investigating the feasibility and effectiveness of combined exercise approaches for PHP, which should be addressed in future research [49, 50]. Given the similar aetiology of PHP and Achilles tendinopathy, and that strength impairments in the plantar fascia are common in PHP, plyometric and kinetic chain strength exercise may have complimentary effects to HSRT in PHP, and improve rehabilitation outcomes, particularly in running athletes. However, the safety, feasibility, and effectiveness of adding plyometric exercise such as jumping or hopping exercise to progressive HSRT, kinetic chain strengthening and education in PHP has not been investigated [51].

A plethora of individual factors have been identified as playing a role in the development and progression of tendinopathy and in individual response to treatment [52]. However, pre-determined exercise rehabilitation protocols do not account for individual training response and as such the potential for strength gains may be greater than this model facilitates [53]. The principle of individualization is considered important when designing resistance training interventions, with evidence suggesting that individually tailored training leads to enhanced adaptations [54, 55]. Individualised exercise prescription and progression may better account for the large inter-individual and biological variability that exist in training responses between individuals [56]. Therefore, using a method known as autoregulation within rehabilitation could allow individuals to increase strength at their own capability and can allow constant adjustment training parameters [57]. Autoregulation is a form of training which involves adjusting the variables of resistance training based on an individual's 
personal factors which may impact readiness to train, leading to enhanced adaptations compared to non-flexible standardized training [57]. Although autoregulation methods originated in rehabilitation and have been found effective for increasing strength and hypertrophy gains in athletes, there is a dearth of interventional studies on its application in physiotherapy, with no studies in tendinopathy [58-60].

Recently, a resistance training specific Rating of Perceived Exertion (RPE) scale has been demonstrated to be effective for measuring effort during training, allowing subsequent load adjustment [61]. The scale measures Repetitions in Reserve (RIR), which allows an individual to estimate the amount of additional repetitions remaining at a given load and adjust load accordingly. Although this scale has been validated with weightlifters, its application in musculoskeletal physiotherapy has not been evaluated [61]. Nevertheless, this training specific RIR-based RPE scale is a potential method to autoregulate training load in patients prescribed resistance training interventions [62]. The specific RIR based RPE autoregulation model has been found to lead to greater training volume, strength improvements and higher training intensity compared to pre-determined programs in athletes $[63,64]$. It could therefore be a potential strategy to ensure that an optimal amount of load is achieved and progressed for each patient in PHP rehabilitation, which is currently not being achieved [38]. A common issue with pre-determined HSRT in tendinopathy is that prescription and progression of loaded sets and repetitions are often poorly defined, with desired training loads not being achieved [38]. If total training dosage is not high enough, then it may not be adequate to stimulate healing through mechanotherapy, resulting in poor outcomes [65]. Previous studies have shown an association between increased exercise dose and recovery for musculoskeletal disorders [36, 66]. An alternative approach to increase exercise dosage may be to increase self-efficacy by empowering patients to be in control of their own rehabilitation, with autoregulation being a potential option to achieve this [67]. 


\section{Methodology}

\subsection{Research question}

The central question this research will ask is: Is it feasible and acceptable to combine autoregulated resistance training and plyometric exercise in rehabilitation using autoregulation progression methods for PHP in running athletes?

\subsection{Aims and objectives}

The primary aim of this study is to test feasibility of the proposed methods, testing recruitment, retention, acceptability, and process evaluation prior to conducting a larger-scale intervention. This will allow understanding of what worked and why in the intervention implementation and contribute to interpretation of preliminary effectiveness. This method of feasibility testing and process evaluation prior to a larger-scale intervention study follows the Medical Research Council's (MRC) recommendations for developing and evaluating complex interventions and should provide insights to aid implementation [68,69]. A secondary aim is to undertake a preliminary analysis of patient-reported outcome measures based on heel pain and function, and to estimate preliminary effectiveness and the number of participants required to adequately power a larger-scale study.

\section{Methods}

\subsection{Study design}

This study will be an explanatory sequential mixed methods feasibility study, testing recruitment, retention, acceptability, and process evaluation of a largerscale definitive study. The quantitative arm is a single centre feasibility prospective cohort study, using a combined resistance training intervention 
progressed with patient autoregulation methods. Outcome measures will be collected at baseline and two periods of post-intervention assessment: 3-months and 6-months follow-up from baseline. The feasibility cohort study will be followed by a qualitative study consisting of semi-structured interviews immediately after intervention completion. The 12-week intervention period has been chosen as it is the same timeframe used in the previous exercise interventions for tendinopathies and is recommended to allow enough time for physiological adaptations from resistance training to occur [36].

\subsection{Location and population}

The clinical site will be a private practice physiotherapy clinic based in the United Kingdom. Participant recruitment will begin from the public, including advertising via social media and local council services, and through contacting local sports centers, teams and running clubs to target running athletes. Additional recruitment sources if required will include contacting local private physiotherapy clinics, including those who specialize in sports injuries. Eligible participants who express interest in taking part will be posted study information materials. The primary investigator and qualified physiotherapist will perform telephone screenings of potentially eligible participants, and those not excluded based on exclusion criteria will be invited to a clinical examination where final eligibility will be determined through ensuring correct diagnosis of PHP and study consent. Consenting participants will also complete outcome measures to establish baseline pain, function, and quality of life (QoL). Demographic and personal information will also be collected at this time. After baseline measures are completed patients will be instructed and guided through the exercise program and progression methods. Selected outcome measures will be repeated at 3-month and 6-month follow-up via postal delivery. Electronic and postal reminders will be sent to participants to help ensure outcome measure completion. 


\subsection{Sample size}

As this is a feasibility study primarily testing study procedures and acceptability, a formal sample size calculation is not required [70]. The minimum target sample size per intervention group is 12 participants for pilot studies, based on recommendations [71]. However, feasibility cohort studies investigating exercises interventions for tendinopathy suggest aiming to recruit 15 participants to adequately assess feasibility outcomes [72]. To help ensure this target is met, the study will aim to recruit $20 \%$ above this target, which is 18 participants in the intervention, allowing for potential dropouts [73]. This should give robust and useful information to inform a sample size calculation for a future trial [70].

\subsection{Inclusion and Exclusion criteria}

The inclusion and exclusion criteria (Table 1) have been developed based on guidelines and current best practice in the literature from recent RCTs investigating the effects of exercise interventions for PHP [36, 38].

\section{Table 1: Inclusion and exclusion criteria}

Inclusion criteria

1. Adults over the age of 18

2. Diagnosis of plantar heel pain by referring clinician to include:

- Heel Pain in the morning or after sitting for extended periods

- Localised pain on palpation where the plantar fascia attaches to the heel - medial calcaneal tuberosity

- Increased pain with walking or standing for extended periods

3. History of at least 3 months of heel pain prior to beginning trial

4. Participates in running or a running sport at least once per week 
5. Signed informed consent form

Exclusion criteria

1. Below 18 years of age

2. Neurological abnormalities, peripheral neuropathy, or nerve entrapment syndromes (e.g., tarsal tunnel syndrome)

3. Systemic inflammatory diseases, rheumatoid arthritis

4. Corticosteroid injection within 3 months of beginning interventions

5. Plantar fascia rupture or previous surgery for heel spur or plantar fascia

6. Pain or stiffness in the first metatarsophalangeal joint to an extent where the strength exercises could not be performed

7. Pregnancy

\subsection{Intervention protocol}

The research physiotherapist will provide instruction and guidance to participants on the resistance training intervention at the clinical site. The researcher will interact with study participants strictly in a standardized way with all participants to reduce any potential confounding variables $[74,75]$. Details of the clinical assessment and the exercise instruction will be reported on standardized forms. The exercise protocol (Table 2) will be based on a combination of autoregulated PFSS, HSRT, plyometric and running exercise and will be performed like that recommended in previous studies [32, 36, 72]. However, the HSRT and plyometric exercises will be progressed using autoregulation methods, with a specific RIR scale detailing load progression based on training response. The research physiotherapist will first instruct and then evaluate each participant to ensure they are performing the exercises correctly at the first session. Exercise technique and understanding of autoregulation progression and pain-monitoring will be evaluated by the research physiotherapist to ensure correct exercise technique and progression to the next stage of exercise. 
Participants will undertake a 12-week progressive exercise program, with four levels of exercise. While pain-monitoring will be used to determine progression throughout the levels, participant autoregulation will be used with the HSRT exercises to individually progress load. Each level will be progressively more challenging and likely to initially provoke symptoms due to increased loading, with participants starting at the level they can undertake with acceptable pain provocation. The exercise program is shown in table 3 and participants will be instructed and given written resources on progressions and regressions. Level 1 will include PFSS and double leg HSRT exercises, progressing to single leg in Level 2 , with double leg jumping added in Level 3 and single leg hopping and running in Level 4. Participants will be educated on the concept of load tolerance and progressive loading, with pain up to $3 / 10$ on the numeric rating scale deemed appropriate during exercise. However acceptable pain levels will be determined individually, to account for individual factors related to pain experience. Patients will be instructed to perform the PFSS while sitting and by first crossing the affected leg over the contralateral leg. Then, while using the hand of the affected side, they are to place the fingers across the base of the toes on the sole of the foot and pull the toes back toward the shin until they felt a stretch in the arch of the foot [32]. Patients are instructed to hold each stretch for a count of ten and to repeat the exercise ten times. They are asked to perform the stretching program at least three times per day, with the first stretch to be done before taking the first step in the morning. In the case of bilateral pain, they were instructed to perform the plantar-specific stretching on both feet [32].

Heavy slow resistance training will consist of unilateral heel raises with a towel inserted under the toes to further activate the windlass mechanism [36]. Participants will be instructed to do the exercise on a stairway, step, or similar location. The towel is individualized, ensuring participants have their toes maximally dorsal flexed at the top of the heel rise. Participants are instructed to perform the exercise every second day for 12 weeks. Every heel rise consists of a 3-second concentric phase (going up) and a 3-second eccentric phase (coming down) with a 2-second isometric phase (pause at the top of the exercise). The HSRT will be progressed individually throughout the trial starting with an 8repetition maximum (RM) for five sets [36]. This is defined as the maximal amount 
of weight that the patient can lift 8 times through the full range of motion while maintaining proper form [38]. If participants cannot perform the required number of repetitions, they are instructed to start the exercises using both legs until they are strong enough to perform unilateral heel raises. They are instructed to keep adding weight to a backpack as they become stronger and to record in their diary the amount used, and number completed. In the case of bilateral pain, they are instructed to perform the heel raise with both limbs [36]. A single kinetic chain HSRT exercise will also be used instead of multiple kinetic chain exercises, to help improve exercise adherence [76]. The single leg squat has been chosen as it simultaneous requires high activation of the gluteus Medius and maximus as demonstrated by electromyography studies [77]. The exercise will be progressed using the same methods and parameters as the HSRT heel-raise. A single exercise intervention based on a progressive single leg squat has been shown to be an effective intervention for patellofemoral pain due to strengthening of the gluteal muscles, which are often weak in PHP patients [78]. If unable to perform a single leg squat, a double leg squat will be included as a regression. The specific autoregulation method used to progress the HSRT exercises will be the RIR scale, with progression criteria detailed in table 2 below.

Level 3 of the exercise program introduces a double leg jumping progression, which is substituted for a single leg hopping and return to running progression in level 4. This progressive plyometric program is based on previous plyometric rehabilitation programs in the tendinopathy literature which have been implemented successfully $[43,79,80]$. Each plyometric stage consists of four different exercises with progressively increasing difficulty. Each stage is progressed and completed at a gradually increased speed when the participant completes each level twice with no increase in pain. In level 4, participants are advised to run for a duration that would not lead to increased pain, and progress with a maximum $10 \%$ increase in volume per week [72].

Low exercise adherence is a common physiotherapy barrier and using a small number of exercises has been shown to result in improved; monitoring, timesaving, self-efficacy, self-management and adherence [67, 76, 81-84]. The 
exercise interventions will be delivered by the research physiotherapist at the clinical site at the first visit, with participants continuing them at home over 12 weeks. The researcher will contact participants either by email or telephone every two weeks to help with any aspect of implementing the exercise intervention or education. Written exercise instruction will be given to participants, including pictures and video links of the exercises together with a description of progression. Participants will also be asked to keep an electronic or written exercise diary to monitor adherence. Participants will also be asked to record in the electronic diary the intake of any pain medication during the study, the number of tablets taken, and the day on which the tablets were taken, as well as any other kind of medication taken during the study. Participants will be asked to use paracetamol within recommended guidelines if pain levels are unacceptable. Consistent with current tendinopathy recommendations, participants will receive an advice and education sheet on; PHP pathology, pain management, medication, activity modification, and how and when to return slowly to activity and running [85].

The participants will receive physiotherapist guidance and exercise instruction along with written instruction. The research physiotherapist will ensure each participant is performing the exercises correctly and answer any questions they have regarding them. Participants will be informed that increased heel pain could occur following exercise, particularly during the first few weeks. The only cointervention to the progressive exercise program is the written educational resource. All other forms of active physiotherapy for PHP will be discouraged but not restricted. Those already wearing orthotics can continue doing so, but new or alteration of orthotics will not be permitted during the study. Participants will also be encouraged not to change their regular shoe wear or activity level during the study. Although there will be no strict restrictions to other physical activity during the study, participants will be encouraged to avoid sudden changes in running volume or intensity, such as starting sprinting or sport suddenly. 
Table 2: Load adjustment during autoregulated resistance training based of rating of percieved exertion and repeitions in reserve vales.

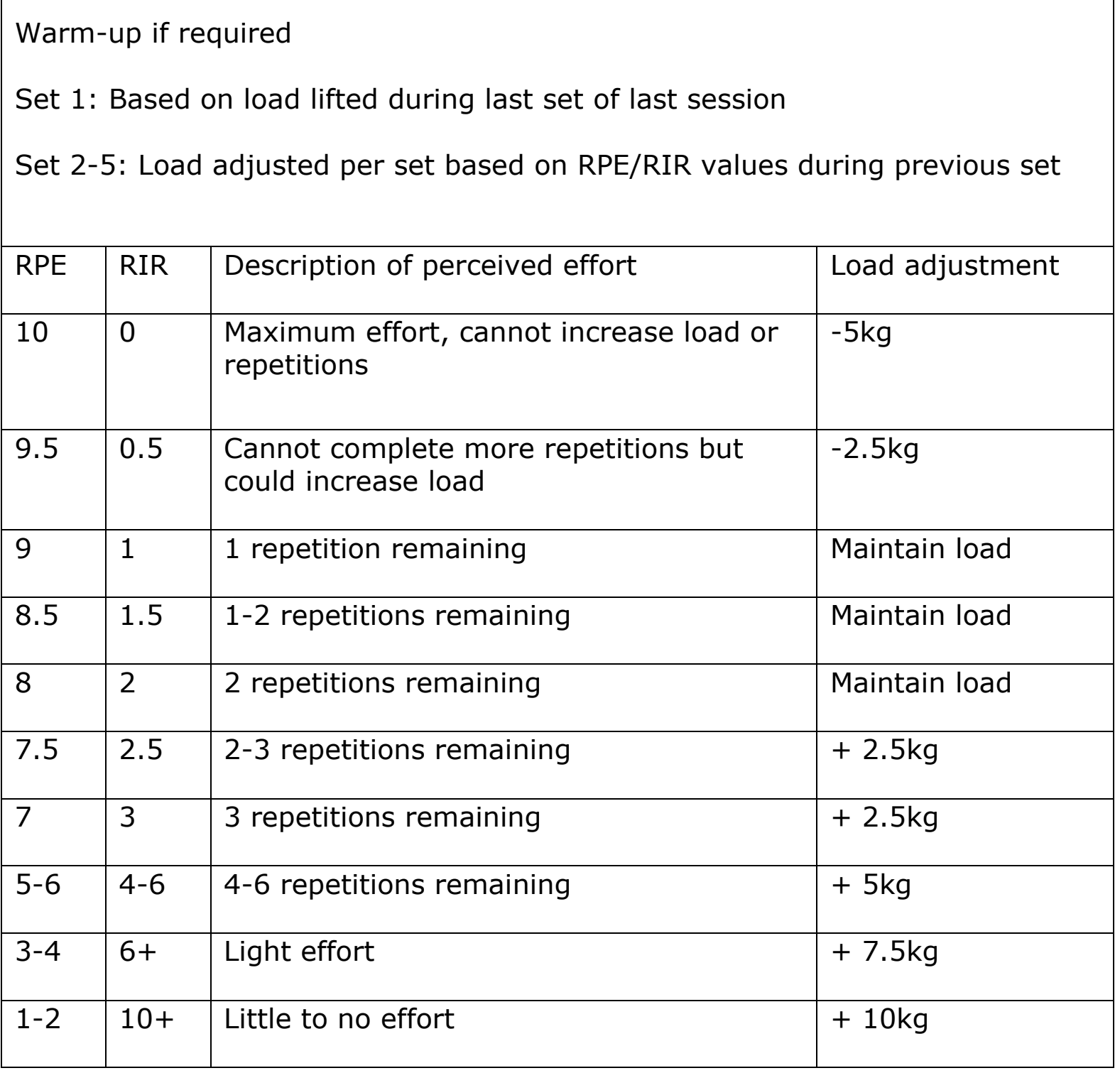

Abbreviations: RPE: rating of perceived exertion; RIR: repetitions in reserve; $\mathrm{Kg}$ : Kilograms. 
Table 3: Progressive autoregulated exercise program (Adapted from Sancho et al. 2019)

\begin{tabular}{|c|c|c|c|c|}
\hline $\begin{array}{l}\text { Level } 1 \\
\text { Pain }>3 / 10 \text { on } \\
\text { NRS as guide. } \\
\text { Patient } \\
\text { acceptable } \\
\text { pain level } \\
\text { during } \\
\text { exercise. }\end{array}$ & $\begin{array}{l}\text { PFSS (daily if } \\
\text { required) } \\
10 \text { times for } \\
10 \text { seconds, } 3 \\
\text { times per day }\end{array}$ & $\begin{array}{l}\text { HSRT (3 } \quad x \\
\text { week) } \\
\text { DLHR (5x8 } \\
\text { repetitions) } \\
\text { DLS (5x8 } \\
\text { repetitions) }\end{array}$ & & \\
\hline $\begin{array}{l}\text { Level } 2 \\
\text { Pain }>3 / 10 \text { on } \\
\text { NRS as guide. } \\
\text { Patient } \\
\text { acceptable } \\
\text { pain level } \\
\text { during } \\
\text { exercise. }\end{array}$ & $\begin{array}{l}\text { PFSS (daily if } \\
\text { required) } \\
10 \text { times for } \\
10 \text { seconds, } 3 \\
\text { times per day }\end{array}$ & 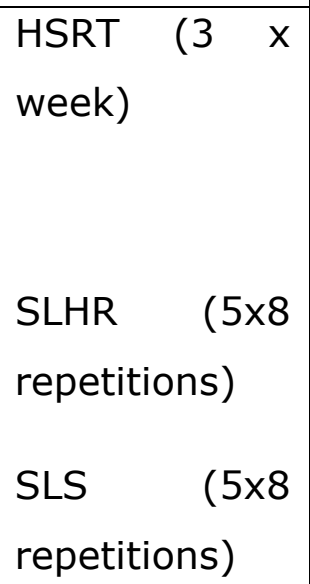 & & \\
\hline $\begin{array}{l}\text { Level } 3 \\
\text { Pain }>3 / 10 \text { on } \\
\text { NRS as guide. } \\
\text { Patient } \\
\text { acceptable } \\
\text { pain level } \\
\text { during } \\
\text { exercise. }\end{array}$ & $\begin{array}{l}\text { PFSS (daily if } \\
\text { required) } \\
10 \text { times for } \\
10 \text { seconds, } 3 \\
\text { times per day }\end{array}$ & 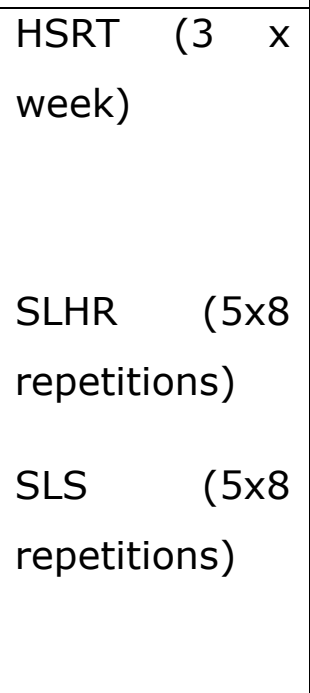 & $\begin{array}{l}\text { DL Jumps ( } 3 \times \\
\text { week) } \\
3 \times 60 \text { DL jump } \\
3 \times 60 \quad \text { stiff } \\
\text { knee } \\
3 \times 5 \\
\text { forward/back } \\
3 \times 5 \text { onto step }\end{array}$ & \\
\hline $\begin{array}{l}\text { Level } 4 \\
\text { Pain }>3 / 10 \text { on }\end{array}$ & $\begin{array}{l}\text { PFSS (daily if } \\
\text { required) }\end{array}$ & $\begin{array}{lll}\text { HSRT } & \left(\begin{array}{ll}3 & x \\
\text { week }\end{array}\right. & \\
\end{array}$ & $\begin{array}{l}\text { SL Hops ( } 1 \text { x } \\
\text { week) }\end{array}$ & $\begin{array}{l}\text { Run (2 } \quad \text { ( } \\
\text { week) } \\
\text { Warm up with }\end{array}$ \\
\hline
\end{tabular}




\begin{tabular}{|c|c|c|c|c|}
\hline $\begin{array}{l}\text { NRS as guide. } \\
\text { Patient } \\
\text { acceptable } \\
\text { pain level } \\
\text { during } \\
\text { exercise. }\end{array}$ & $\begin{array}{l}10 \text { times for } \\
10 \text { seconds, } 3 \\
\text { times per day }\end{array}$ & $\begin{array}{l}\text { SLHR } \quad(5 \times 8 \\
\text { repetitions) } \\
\text { SLS (5x8 } \\
\text { repetitions) }\end{array}$ & $\begin{array}{l}3 \times 60 \text { DL jump } \\
3 \times 30 \text { SL hop } \\
3 \times 15 \text { stiff } \\
\text { knee } \\
3 \times 5 \\
\text { forward/back } \\
3 \times 10 \quad \text { onto } \\
\text { step }\end{array}$ & $\begin{array}{l}\text { DL jumps and } \\
\text { SL hops. Slow } \\
\text { pace, } 10 \% \\
\text { max volume } \\
\text { increase per } \\
\text { week }\end{array}$ \\
\hline
\end{tabular}

Abbreviations: NRS: numeric rating scale; HSRT: heavy slow resistance training; PFSS; plantar-fascia specific stretching; DL: double leg; SL: single leg; SLS: single leg squat; HR: heel raise; SLHR: single-leg heel raise; DLS: double leg squat.

\subsection{Outcome measures}

Feasibility and acceptability of study intervention procedures and process evaluation outcome data will be the primary outcomes measured to determine feasibility of a definitive study, large enough to determine treatment effects [68, 69]. Analysing process evaluation data prior to outcome analysis is recommended to avoid biased interpretation of process data [86]. Data will be collected on recruitment, retention, outcome measure response rates, adverse events, exercise adherence, intervention acceptability and fidelity [87]. Acceptability of outcome measures and the interventions will be measured by questionnaires and the interviews following the intervention. Adherence to the exercise program will be measured by a training diary given to each participant at baseline to record exercises completed and progressions [38]. Participants will be instructed on how to correctly record the number of repetitions, sets and progressions and the days on which they performed them. Implementation fidelity will be assessed by recording attendance, exercise adherence, intervention checklists and participant diaries and interviews [88]. Implementation fidelity can be defined as the degree to which an intervention is implemented as intended $[89,90]$. Fidelity enhances 
scientific confidence that study outcomes are due to interventions and not implementation variables [91-93]. Fidelity remains poorly reported in physiotherapy research, with a need for robust protocols to ensure clinical translation from findings [94-97].

Secondary outcome measures have been selected based on applicability, guidelines, psychometric properties, and previous research for PHP patients undergoing physiotherapy interventions. These patient-reported outcome measures will be completed at baseline assessment and at 3-month and 6-month follow-up electronically, with participants receiving electronic and postal completion reminders. Pain visual analogue scale (VAS) scores is a standard outcome measures used in physiotherapy to measure pain and has good reliability for musculoskeletal disorders; however, it has questionable validity [98]. A change of $9 \mathrm{~mm}$ on a $100 \mathrm{~mm}$ scale is considered clinically relevant for patients with PHP [47]. The EuroQol 5-dimension (EQ-5D-5L) is a common global health questionnaire with good validity when tested across several populations to measure quality of life, it is also recommended for use by the NHS $[99,100]$.

The Foot Function Index (FFI) is a common foot specific validated outcome measure used in practice for PHP with good psychometric properties [101-103]. The FFI is recommended by several bodies as a validated physiotherapy PHP outcome measure, such as the American physical therapy association [104]. The FFI consists of 23 self-reported items divided into 3 subcategories based on patient values: pain, disability, and activity limitation. [105]. The minimal important difference is 7 points for the total scale in PHP interventions [106]. The Foot and Ankle Ability Measure (FAAM) is a 29-item questionnaire divided into two subscales: 21-item Activities of Daily Living ( $A D L$ ) Subscale and the, 8-item Sports Subscale. The Sports subscale assesses more difficult tasks that are essential to sport and is a population-specific subscale designed for athletes [107]. The minimal clinically important differences are 8 and 9 points for the ADL and Sports subscales, respectively [108]. The FAAM is the only outcome measure which has been validated in a physiotherapy setting specifically with the PHP population [109]. The FAAM has been validated for test content, internal 
structure, score stability and responsiveness, with Test-retest reliability of 0.89 and 0.87 for the ADL and sports subscales [110]. Evidence for content validity, construct validity, reliability, and responsiveness were obtained among 243 subjects who were separated into a group expected to change and a group expected to remain stable [108]. Although both the FFI and FAAM are recommended outcome measures for PHP, the FAAM is a more appropriate measure to use with athletic individuals [104].

\section{Table 4: Secondary outcome measures}

\section{Secondary outcome measures assessing pain, function and QoL}

Foot Function Index (FFI)

Foot and Ankle Ability Measure (FAAM) score

Visual analogue scale (VAS) pain scores on $100 \mathrm{~mm}$ scale

EQ-5D-5L QoL score

\subsection{Data processing and analysis}

Outcome measures will be completed on paper or electronically by participants then transferred to excel and SPSS for analysis by the researcher. Quantitative data about the intervention implementation will be analysed using descriptive statistics. Data will be collected on recruitment rates, retention, adherence, compliance, fidelity, drop-outs, and secondary outcome measure completion. Data will be analysed as percentages for categorical data and mean standard deviation for continuous data. Data findings will determine process evaluation and intervention procedures of a larger-scale study. Exploratory inferential analyses of the secondary outcome measures will allow calculation of effect sizes to produce sample size calculations required for a larger-scale study. 


\subsection{Qualitative interviews}

Following intervention completion, selected participants will undergo semistructured telephone interviews to cover issues related to trial procedures and acceptability. The interviews will have two aims; to determine patients' acceptability of the interventions and gain insight to participants views and perceptions on resistance training and autoregulation methods for PHP treatment for the first time in the literature. Semi-structured interviews allow participants to express their views and experiences in rich detail, while maintaining some structure to address the study objectives [111]. Although the absence of visual cues via telephone is thought to limit non-verbal data and compromise rapport, there is a lack of evidence showing they produce lower-quality data [112]. Participants may also feel more relaxed and comfortable disclosing information via telephone allowing rich narrative data collection [113].

Purposive sampling will be used to select an estimated 12 participants based on sampling criteria of age and gender to ensure a broad range of perspectives [114] Purposive sampling along dimensions expected to influence complex interventions is recommended for qualitative interviews within process evaluations [115]. Acceptability to outcome measure burden, the interventions and trial participation will be assessed. Personal and contextual factors which may affect outcomes will also be explored, such as barriers, intervention expectations and acceptability [116]. Collecting qualitative data can also help in identifying barriers, implementation strategies and acceptable treatment fidelity protocols for a larger scale study [117]. An example of the proposed interview question format is provided in table 5. Interviews will be recorded using a digital voice recorder, fully anonymised and transcribed verbatim [118]. Data will be analysed using the framework method of analysis involving data familiarisation, identifying important themes and issues to develop a thematic framework [111]. Data will be indexed according to the framework leading to a charting of data according to the thematic framework. Mapping and interpreting the themes will help to clarify concepts and help understand and explain the data in relation to the study objectives. 


\section{Table 5: Semi-structured interview topic guide}

Interview topic guide

1. Introduction

- Introduction of interviewer and overview of study

- Discussion of consent, confidentiality, and interview procedure

- Check if interviewee has any questions and happy to proceed Switch on voice recorder

- Record verbal consent to each item on consent form

2. Background factors

- Length of time with plantar heel pain (PHP)

- The type of physical activities interviewees performs

- In what ways does PHP present as a problem

- What previous PHP treatments have they tried?

- What ways have other treatments helped

3. Preconceptions and expectations of exercise for PHP

- What knowledge do participants have about exercise?

- What do participants understand about how exercise works for PHP?

- What do participants understand about the exercise procedure?

- Were there any side-effects from exercise?

- What did participants expect would be the outcome from exercise for PHP?

- Short and long-term outcomes?

- What do participants personally consider a successful outcome?

4. Treatment Experiences of exercise

- What were participants told about exercise for PHP from healthcare professionals 
- Did this change what they initially understood and expected from exercise for PHP

- What were participants first experiences of exercise for PHP like?

- What were the positive/negative effects of this experience?

- Was the experience what participants expected?

- If so, why? If not, why not?

- What was done well during the treatment and what could have been done better?

- What are participants current views about exercise after treatment

- Is the treatment relevant to the participants PHP condition?

- Perceived outcomes from exercise treatment (short and long-term)

5. Process evaluation $\&$ trial procedures

- Did you find the outcome measures and time taken to complete them acceptable?

- Did you find the recruitment $\&$ assessment process acceptable?

- What was your clinical treatment experience like?

- What are your views on the provided education \& advice information?

- What are your views on the exercise program?

- What are your views on the autoregulation self-progression methods?

- Did you receive enough guidance and written instruction to allow you to complete the exercise program at home? Could this have been provided in a more helpful manner?

- Could anything about your study experience be improved?

6. Close interview

- Summarise discussion

- Reiterate confidentially

- Invite any questions

- Thank participants 


\subsection{Ethical issues}

When conducting clinical research certain ethical issues should be anticipated, including informed consent, or autonomy and freedom of choice to participate [119]. Researchers must also take steps to mitigate harm to participants such as ensuring they are fully aware of any risks, the study purpose and are not deceived [120]. Research must also benefit participants, such as not withholding treatment in experimental intervention studies [121]. To achieve this, the proposed study group is receiving an exercise treatment that is hypothesised to be effective for PHP. Recruited participants will be informed of the study purpose, potential risks, and provided written informed consent. With resistance and plyometric exercise, there is a small risk of temporarily increased pain, which patients' will be informed about [28]. Another key ethical issue is right to privacy and confidentiality, attention must be paid to how information is obtained, stored, processed, and disseminated [122]. Confidentiality risk can be higher in qualitative studies using direct quotes, particularly those involving small specialized sub-populations with a specific pathology in one geographical region [123]. All quantitative and qualitative data collected will be securely stored on research drives accessed via password-protected computers in accordance with the Data protection Act (1998). Confidentiality for participants will be ensured throughout the project and all participants will be assigned an ID code. Data will therefore be re-identifiable; however, the code sheet will always be stored separately from participant data. The authors for this study have no conflicts of interest or sources of external funding. Other ethical issues to consider are researcher competency, study design and worthwhile expected outcomes [124]. By employing exercise treatments with high-quality evidence supporting them, this study expects to achieve positive outcomes for patients. Ethics committees expect research to be of high methodological quality and address unanswered questions [119]. The proposed study aims to employ rigorous methodological design and aims to investigate questions regarding feasibility and acceptability of exercise and autoregulation progression methods in PHP. The study authors will also apply for the required ethical approval, with the research physiotherapist having attended NHS research training. 


\section{Limitations and potential risks}

Despite the high prevalence of PHP there may be a lack of participants available or willing to take part during the recruitment time phase. Low recruitment is high risk because a minimum of 15 participants is required for the feasibility cohort study intervention group to gather robust data. The population being recruited is also highly specific, in that they must have PHP and be involved in regular running activities. In the event of low recruitment levels, the researcher could meet with recruitment sources such as local sports clubs to identify any barriers and offer strategies for increasing recruitment. Alternative recruitment methods could also be considered such as recruiting from the NHS, private hospitals, physiotherapy, or sports injuries clinics in the area. As follow-up outcome measure collection will be via postal delivery there is a risk that participants will not return these measures. Low response rates may affect the accuracy and robustness of study findings. Sending reminders to return outcome measures would be a strategy to prevent this, by initially automatically emailing or text messaging participants to remind them and then phoning participants if they have not returned them by the requested date. Providing the option to complete outcome measures electronically could also reduce this risk, due to lower participant burden. Participants for interviews will be selected purposively by age and gender, with an anticipated 12 total participants out of the target recruitment of 18 required. However, there is a risk that selected participants will decline to participate. Interview participation could therefore be offered to those not purposively selected.

\section{Originality and impact of the research}

Musculoskeletal disorders such as PHP are having an increasing burden on society. Recently HSRT has emerged as an effective PHP treatment, however more comprehensive exercise protocols and progression methods are needed, as longterm outcomes remain poor. Plyometric exercise is often discouraged in PHP but has not been investigated as a potential rehabilitation component in PHP, despite being found feasible in Achilles tendinopathy. It could be hypothesised that 
combining HSRT and plyometric exercise could lead to superior outcomes for PHP. In clinical practice, multiple types of exercise are commonly prescribed, and it is recommended that PHP not be treated by one exercise type in isolation. However, intervention studies most often use one type of exercise in isolation, with there being a need to investigate feasibility and effectiveness of comprehensive exercise programs in PHP. Therefore, this feasibility study investigating a comprehensive exercise program for PHP would be the first in the literature to determine if combined exercise treatment is feasible and potentially effective. Determining feasibility would have direct implications on clinical practice, allowing recommendations to be made, which are currently lacking.

Rehabilitation programs in PHP are currently not achieving required training intensity and dosage with better methods for progressing resistance training required. One potential method is autoregulation, which allows individuals to progress at their own rate, taking individual factors into account. Specific autoregulation methods such as the RPE RIR scale have been shown to be more effective to increase strength compared to pre-determined programs in athletes but have not been investigated in rehabilitation. This feasibility study would help determine if the RPE RIR autoregulation method is feasible and acceptable for use in an athletic PHP population. Patients expectations, and views on feasibility and acceptability of different types of exercise as well as autoregulation methods of progression for PHP are unknown. Therefore, the addition of qualitative interviews in a mixed methods format would help ascertain acceptability of these methods and exercise interventions for the first time and help explain the intervention outcomes in the cohort study, allowing for design of a larger-scale study. The combination of these different study types would allow for collection of original research data not explored before, allowing the potential to make a significant contribution to the PHP rehabilitation literature. 
Funding: No sources of funding were used to assist in the preparation of this article.

Conflicts of interest/Competing interests: The author declares no conflicts of interest relevant to the content of this review.

Authorship contributions: IB conceptualised the work, wrote the first draft of the manuscript, revised the manuscript and approved the final manuscript.

\section{References}

1. Briggs AM, Woolf AD, Dreinhofer K, Homb N, Hoy DG, Kopansky-Giles D, et al. Reducing the global burden of musculoskeletal conditions. Bull World Health Organ. 2018 May 1;96(5):366-8.

2. Hoy DG, Smith E, Cross M, Sanchez-Riera L, Blyth FM, Buchbinder R, et al. Reflecting on the global burden of musculoskeletal conditions: Lessons learnt from the global burden of disease 2010 study and the next steps forward. Ann Rheum Dis. 2015 Jan;74(1):4-7.

3. Scarborough P, Bhatnagar P, Wickramasinghe KK, Allender S, Foster C, Rayner M. The economic burden of ill health due to diet, physical inactivity, smoking, alcohol and obesity in the UK: An update to 2006-07 NHS costs. J Public Health (Oxf). 2011 Dec;33(4):527-35.

4. Yelin E, Weinstein S, King T. An update on the burden of musculoskeletal diseases in the U.S. Semin Arthritis Rheum. 2019 Aug;49(1):1-2.

5. Trojian T, Tucker AK. Plantar fasciitis. Am Fam Physician. 2019 06/15;99(12):744-50. 
6. Monteagudo M, de Albornoz PM, Gutierrez B, Tabuenca J, Alvarez I. Plantar fasciopathy: A current concepts review. EFORT Open Rev. 2018 Aug 29;3(8):48593.

7. Riel $H$, Lindstrom CF, Rathleff MS, Jensen MB, Olesen JL. Prevalence and incidence rate of lower-extremity tendinopathies in a danish general practice: A registry-based study. BMC Musculoskelet Disord. 2019 May 22;20(1):239,0192629-6.

8. Rasenberg N, Bierma-Zeinstra SMA, Fuit L, Rathleff MS, Dieker A, van Veldhoven $\mathrm{P}$, et al. Custom insoles versus sham and GP-led usual care in patients with plantar heel pain: Results of the STAP-study - a randomised controlled trial. Br J Sports Med. 2020 Sep 2.

9. Gutteck N, Schilde S, Delank KS. Pain on the plantar surface of the foot. Dtsch Arztebl Int. 2019 Feb 8;116(6):83-8.

10. Hasegawa M, Urits I, Orhurhu V, Orhurhu MS, Brinkman J, Giacomazzi S, et al. Current concepts of minimally invasive treatment options for plantar fasciitis: A comprehensive review. Curr Pain Headache Rep. 2020 Aug 12;24(9):55,02000883-7.

11. Schuitema D, Greve C, Postema K, Dekker R, Hijmans JM. Effectiveness of mechanical treatment for plantar fasciitis: A systematic review. J Sport Rehabil. 2019 Oct 18:1-18.

12. Lopes $A D$, Hespanhol Junior LC, Yeung SS, Costa LO. What are the main running-related musculoskeletal injuries? A systematic review. Sports Med. 2012 Oct $1 ; 42(10): 891-905$.

13. Orchard J. Plantar fasciitis. BMJ. 2012 Oct 10;345:e6603.

14. Murphy K, Curry EJ, Matzkin EG. Barefoot running: Does it prevent injuries? Sports Med. 2013 Nov;43(11):1131-8.

15. Bolgla LA, Malone TR. Plantar fasciitis and the windlass mechanism: A biomechanical link to clinical practice. J Athl Train. 2004 Jan;39(1):77-82. 
16. Ribeiro AP, Joao SM, Dinato RC, Tessutti VD, Sacco IC. Dynamic patterns of forces and loading rate in runners with unilateral plantar fasciitis: A cross-sectional study. PLoS One. 2015 Sep 16;10(9):e0136971.

17. Wu CH, Chang KV, Mio S, Chen WS, Wang TG. Sonoelastography of the plantar fascia. Radiology. 2011 May;259(2):502-7.

18. Goff JD, Crawford R. Diagnosis and treatment of plantar fasciitis. Am Fam Physician. 2011 Sep 15;84(6):676-82.

19. Petraglia F, Ramazzina I, Costantino C. Plantar fasciitis in athletes: Diagnostic and treatment strategies. A systematic review. Muscles Ligaments Tendons J. 2017 May $10 ; 7(1): 107-18$.

20. Salvioli S, Guidi M, Marcotulli G. The effectiveness of conservative, nonpharmacological treatment, of plantar heel pain: A systematic review with metaanalysis. Foot (Edinb). 2017 Dec;33:57-67.

21. Fraser J], Corbett R, Donner C, Hertel J. Does manual therapy improve pain and function in patients with plantar fasciitis? A systematic review. J Man Manip Ther. 2018 May;26(2):55-65.

22. Fraser JJ, Glaviano NR, Hertel J. Utilization of physical therapy intervention among patients with plantar fasciitis in the united states. J Orthop Sports Phys Ther. 2017 Feb;47(2):49-55.

23. Grieve R, Palmer S. Physiotherapy for plantar fasciitis: A UK-wide survey of current practice. Physiotherapy. 2017 Jun;103(2):193-200.

24. Sussman WI, Mautner K, Malanga G. The role of rehabilitation after regenerative and orthobiologic procedures for the treatment of tendinopathy: $A$ systematic review. Regen Med. 2018 Mar;13(2):249-63.

25. Babatunde OO, Legha A, Littlewood C, Chesterton LS, Thomas MJ, Menz HB, et al. Comparative effectiveness of treatment options for plantar heel pain: $A$ systematic review with network meta-analysis. $\mathrm{Br}$ J Sports Med. 2019 $02 ; 53(3): 182-94$. 
26. Shetty SH, Dhond A, Arora M, Deore S. Platelet-rich plasma has better longterm results than corticosteroids or placebo for chronic plantar fasciitis: Randomized control trial. J Foot Ankle Surg. 2019 Jan;58(1):42-6.

27. Whittaker GA, Munteanu SE, Menz HB, Bonanno DR, Gerrard JM, Landorf KB. Corticosteroid injection for plantar heel pain: A systematic review and metaanalysis. BMC Musculoskelet Disord. 2019 Aug 17;20(1):378,019-2749-z.

28. Roerdink RL, Dietvorst $M$, van der Zwaard B, van der Worp $H$, Zwerver J. Complications of extracorporeal shockwave therapy in plantar fasciitis: Systematic review. Int J Surg. 2017 Oct;46:133-45.

29. Thomas MJ, Whittle R, Menz HB, Rathod-Mistry T, Marshall M, Roddy E. Plantar heel pain in middle-aged and older adults: Population prevalence, associations with health status and lifestyle factors, and frequency of healthcare use. BMC Musculoskelet Disord. 2019 Jul 20;20(1):337,019-2718-6.

30. Jimenez-Perez AE, Gonzalez-Arabio D, Diaz AS, Maderuelo JA, Ramos-Pascua LR. Clinical and imaging effects of corticosteroids and platelet-rich plasma for the treatment of chronic plantar fasciitis: A comparative non randomized prospective study. Foot Ankle Surg. 2019 Jun;25(3):354-60.

31. DiGiovanni BF, Moore AM, Zlotnicki JP, Pinney SJ. Preferred management of recalcitrant plantar fasciitis among orthopaedic foot and ankle surgeons. Foot Ankle Int. 2012 Jun;33(6):507-12.

32. . DiGiovanni BF, Nawoczenski DA, Lintal ME, Moore EA, Murray JC, Wilding GE, et al. Tissue-specific plantar fascia-stretching exercise enhances outcomes in patients with chronic heel pain. A prospective, randomized study. J Bone Joint Surg Am. 2003 Jul;85-A(7):1270-7.

33. Digiovanni BF, Nawoczenski DA, Malay DP, Graci PA, Williams TT, Wilding GE, et al. Plantar fascia-specific stretching exercise improves outcomes in patients with chronic plantar fasciitis. A prospective clinical trial with two-year follow-up. J Bone Joint Surg Am. 2006 Aug;88(8):1775-81. 
34. Caratun R, Rutkowski NA, Finestone HM. Stubborn heel pain: Treatment of plantar fasciitis using high-load strength training. Can Fam Physician. 2018 Jan;64(1):44-6.

35. Engkananuwat $\mathrm{P}$, Kanlayanaphotporn $\mathrm{R}$, Purepong $\mathrm{N}$. Effectiveness of the simultaneous stretching of the achilles tendon and plantar fascia in individuals with plantar fasciitis. Foot Ankle Int. 2018 Jan;39(1):75-82.

36. Rathleff MS, Molgaard CM, Fredberg U, Kaalund S, Andersen KB, Jensen TT, et al. High-load strength training improves outcome in patients with plantar fasciitis: A randomized controlled trial with 12-month follow-up. Scand J Med Sci Sports. 2015 Jun;25(3):e292-300.

37. Cil ET, Sayli U, Subasi F. Outpatient vs home management protocol results for plantar fasciitis. Foot Ankle Int. 2019 Aug 6:1071100719865302.

38. Riel H, Jensen MB, Olesen JL, Vicenzino B, Rathleff MS. Self-dosed and predetermined progressive heavy-slow resistance training have similar effects in people with plantar fasciopathy: A randomised trial. J Physiother. 2019 Jul;65(3): 144-51.

39. McAuliffe S, Tabuena A, McCreesh K, O'Keeffe M, Hurley J, Comyns T, et al. Altered strength profile in achilles tendinopathy: A systematic review and metaanalysis. J Athl Train. 2019 Aug;54(8):889-900.

40. Child S, Bryant AL, Clark RA, Crossley KM. Mechanical properties of the achilles tendon aponeurosis are altered in athletes with achilles tendinopathy. Am J Sports Med. 2010 Sep;38(9):1885-93.

41. Gajhede-Knudsen M, Ekstrand J, Magnusson H, Maffulli N. Recurrence of achilles tendon injuries in elite male football players is more common after early return to play: An 11-year follow-up of the UEFA champions league injury study. Br J Sports Med. 2013 Aug;47(12):763-8.

42. Silbernagel KG, Thomee R, Eriksson BI, Karlsson J. Full symptomatic recovery does not ensure full recovery of muscle-tendon function in patients with achilles tendinopathy. Br J Sports Med. 2007 Apr;41(4):276,80; discussion 280. 
43. Silbernagel KG, Thomee R, Eriksson BI, Karlsson J. Continued sports activity, using a pain-monitoring model, during rehabilitation in patients with achilles tendinopathy: A randomized controlled study. Am J Sports Med. 2007 Jun;35(6):897-906.

44. Kamonseki DH, Goncalves GA, Yi LC, Junior IL. Effect of stretching with and without muscle strengthening exercises for the foot and hip in patients with plantar fasciitis: A randomized controlled single-blind clinical trial. Man Ther. 2016 Jun;23:76-82.

45. Sweeting D, Parish B, Hooper L, Chester R. The effectiveness of manual stretching in the treatment of plantar heel pain: A systematic review. J Foot Ankle Res. 2011 Jun 25;4:19,1146-4-19.

46. Almubarak A, Foster N. Exercise therapy for plantar heel pain: A systematic review. International Journal of Exercise Science. $2012 ; 5(3)$.

47. Johannsen FE, Herzog RB, Malmgaard-Clausen NM, Hoegberget-Kalisz M, Magnusson SP, Kjaer M. Corticosteroid injection is the best treatment in plantar fasciitis if combined with controlled training. Knee Surg Sports Traumatol Arthrosc. 2019 Jan;27(1):5-12.

48. Sutton DA, Nordin M, Cote $\mathrm{P}$, Randhawa $K, \mathrm{Yu} \mathrm{H}$, Wong JJ, et al. The effectiveness of multimodal care for soft tissue injuries of the lower extremity: A systematic review by the ontario protocol for traffic injury management (OPTIMa) collaboration. J Manipulative Physiol Ther. 2016 Feb;39(2):95,109.e1-2.

49. Cinar E, Saxena S, Akkurt HE, Uygur F. Extracorporeal shockwave therapy in the management of plantar fasciitis: A randomized controlled trial. Foot (Edinb). 2020 Sep;44:101679.

50. Cinar E, Saxena S, Uygur F. Combination therapy versus exercise and orthotic support in the management of pain in plantar fasciitis: A randomized controlled trial. Foot Ankle Int. 2018 Jan 1:1071100717747590.

51. Huffer D, Hing W, Newton R, Clair M. Strength training for plantar fasciitis and the intrinsic foot musculature: A systematic review. Phys Ther Sport. 2017 Mar;24:44-52. 
52. Steinmann S, Pfeifer CG, Brochhausen C, Docheva D. Spectrum of tendon pathologies: Triggers, trails and end-state. Int J Mol Sci. 2020 Jan 28;21(3):10.3390/ijms21030844.

53. Timmons JA. Variability in training-induced skeletal muscle adaptation. J Appl Physiol (1985). 2011 Mar;110(3):846-53.

54. Jones N, Kiely J, Suraci B, Collins DJ, de Lorenzo D, Pickering C, et al. A genetic-based algorithm for personalized resistance training. Biol Sport. 2016 Jun;33(2):117-26.

55. Borresen J, Lambert MI. The quantification of training load, the training response and the effect on performance. Sports Med. 2009;39(9):779-95.

56. Mann TN, Lamberts RP, Lambert MI. High responders and low responders: Factors associated with individual variation in response to standardized training. Sports Med. 2014 Aug;44(8):1113-24.

57. Mann JB, Thyfault JP, Ivey PA, Sayers SP. The effect of autoregulatory progressive resistance exercise vs. linear periodization on strength improvement in college athletes. J Strength Cond Res. 2010 Jul;24(7):1718-23.

58. Horschig AD, Neff TE, Serrano AJ. Utilization of autoregulatory progressive resistance exercise in transitional rehabilitation periodization of a high school football-player following anterior cruciate ligament reconstruction: A case report. Int J Sports Phys Ther. 2014 Oct;9(5):691-8.

59. Ardali G. A daily adjustable progressive resistance exercise protocol and functional training to increase quadriceps muscle strength and functional performance in an elderly homebound patient following a total knee arthroplasty. Physiother Theory Pract. 2014 May;30(4):287-97.

60. Rauch JT, Ugrinowitsch C, Barakat CI, Alvarez MR, Brummert DL, Aube DW, et al. Auto-regulated exercise selection training regimen produces small increases in lean body mass and maximal strength adaptations in strength-trained individuals. J Strength Cond Res. 2020 Apr;34(4):1133-40. 
61. Zourdos MC, Klemp A, Dolan C, Quiles JM, Schau KA, Jo E, et al. Novel resistance training-specific rating of perceived exertion scale measuring repetitions in reserve. J Strength Cond Res. 2016 Jan;30(1):267-75.

62. Fairman CM, Zourdos MC, Helms ER, Focht BC. A scientific rationale to improve resistance training prescription in exercise oncology. Sports Med. 2017 Aug;47(8): 1457-65.

63. Helms ER, Cross MR, Brown SR, Storey A, Cronin J, Zourdos MC. Rating of perceived exertion as a method of volume autoregulation within a periodized program. J Strength Cond Res. 2018 Jun;32(6):1627-36.

64. Graham T, Cleather DJ. Autoregulation by "repetitions in reserve" leads to greater improvements in strength over a 12-week training program than fixed loading. J Strength Cond Res. 2019 Apr 17.

65. Khan KM, Scott A. Mechanotherapy: How physical therapists' prescription of exercise promotes tissue repair. Br J Sports Med. 2009 Apr;43(4):247-52.

66. Osteras B, Osteras $H$, Torstensen TA, Vasseljen O. Dose-response effects of medical exercise therapy in patients with patellofemoral pain syndrome: $A$ randomised controlled clinical trial. Physiotherapy. 2013 Jun;99(2):126-31.

67. Jack K, McLean SM, Moffett JK, Gardiner E. Barriers to treatment adherence in physiotherapy outpatient clinics: A systematic review. Man Ther. 2010 Jun;15(3):220-8.

68. Craig P, Dieppe P, Macintyre S, Michie S, Nazareth I, Petticrew M, et al. Developing and evaluating complex interventions: The new medical research council guidance. BMJ. 2008 Sep 29;337:a1655.

69. Moore GF, Audrey S, Barker M, Bond L, Bonell C, Hardeman W, et al. Process evaluation of complex interventions: Medical research council guidance. BMJ. 2015 Mar 19;350:h1258.

70. Arain M, Campbell MJ, Cooper CL, Lancaster GA. What is a pilot or feasibility study? A review of current practice and editorial policy. BMC Med Res Methodol. 2010 Jul 16;10:67,2288-10-67. 
71. Lannin NA, Ada L, Levy T, English C, Ratcliffe J, Sindhusake D, et al. Intensive therapy after botulinum toxin in adults with spasticity after stroke versus botulinum toxin alone or therapy alone: A pilot, feasibility randomized trial. Pilot Feasibility Stud. 2018 May 22;4:82,018-0276-6. eCollection 2018.

72. Sancho I, Morrissey D, Willy RW, Barton C, Malliaras P. Education and exercise supplemented by a pain-guided hopping intervention for male recreational runners with midportion achilles tendinopathy: A single cohort feasibility study. Phys Ther Sport. 2019 Nov;40:107-16.

73. Gupta A, Calfas KJ, Marshall SJ, Robinson TN, Rock CL, Huang JS, et al. Clinical trial management of participant recruitment, enrollment, engagement, and retention in the SMART study using a marketing and information technology (MARKIT) model. Contemp Clin Trials. 2015 May;42:185-95.

74. Suttanon P, Hill KD, Said CM, Williams SB, Byrne KN, LoGiudice D, et al. Feasibility, safety and preliminary evidence of the effectiveness of a home-based exercise programme for older people with alzheimer's disease: A pilot randomized controlled trial. Clin Rehabil. 2013 May;27(5):427-38.

75. Giangregorio LM, MacIntyre NJ, Heinonen A, Cheung AM, Wark JD, Shipp K, et al. Too fit to fracture: A consensus on future research priorities in osteoporosis and exercise. Osteoporos Int. 2014 May;25(5):1465-72.

76. McLean SM, Burton M, Bradley L, Littlewood C. Interventions for enhancing adherence with physiotherapy: A systematic review. Man Ther. 2010 Dec; 15(6): 514-21.

77. Boren K, Conrey C, Le Coguic J, Paprocki L, Voight M, Robinson TK. Electromyographic analysis of gluteus medius and gluteus maximus during rehabilitation exercises. Int J Sports Phys Ther. 2011 Sep;6(3):206-23.

78. Drew BT, Conaghan PG, Smith TO, Selfe J, Redmond AC. The effect of targeted treatment on people with patellofemoral pain: A pragmatic, randomised controlled feasibility study. BMC Musculoskelet Disord. 2017 Aug 4;18(1):338,017-1698-7.

79. Kountouris A, Cook J. Rehabilitation of achilles and patellar tendinopathies. Best Pract Res Clin Rheumatol. 2007 Apr;21(2):295-316. 
80. Malliaras P, Cook J, Purdam C, Rio E. Patellar tendinopathy: Clinical diagnosis, load management, and advice for challenging case presentations. J Orthop Sports Phys Ther. 2015 Nov;45(11):887-98.

81. de Vries HJ, Kloek CJJ, de Bakker DH, Dekker J, Bossen D, Veenhof C. Determinants of adherence to the online component of a blended intervention for patients with hip and/or knee osteoarthritis: A mixed methods study embedded in the e-exercise trial. Telemed J E Health. 2017 Dec;23(12):1002-10.

82. Hoffmann TC, Del Mar CB, Strong J, Mai J. Patients' expectations of acute low back pain management: Implications for evidence uptake. BMC Fam Pract. 2013 Jan $8 ; 14: 7,2296-14-7$.

83. Littlewood C, Bateman M, Brown K, Bury J, Mawson S, May S, et al. A selfmanaged single exercise programme versus usual physiotherapy treatment for rotator cuff tendinopathy: A randomised controlled trial (the SELF study). Clin Rehabil. 2016 Jul;30(7):686-96.

84. Willett M, Duda J, Gautrey C, Fenton S, Greig C, Rushton A. Effectiveness of behavioural change techniques in physiotherapy interventions to promote physical activity adherence in patients with hip and knee osteoarthritis: A systematic review protocol. BMJ Open. 2017 Jun 30;7(6):e015833,2017-015833.

85. MARTIN RL, CHIMENTI R, CUDDEFORD T, HOUCK J, MATHESON JW, MCDONOUGH CM, et al. Achilles pain, stiffness, and muscle power deficits: Midportion achilles tendinopathy revision 2018. Journal of Orthopaedic \& Sports Physical Therapy. 2018 05;48(5):A1-A38.

86. Oakley A, Strange V, Bonell C, Allen E, Stephenson J, RIPPLE Study Team. Process evaluation in randomised controlled trials of complex interventions. BMJ. 2006 Feb 18;332(7538):413-6.

87. Littlewood C, Bateman M, Cooke K, Hennnings S, Cookson T, Bromley K, et al. Protocol for a multi-centre pilot and feasibility randomised controlled trial with a nested qualitative study: Rehabilitation following rotator cuff repair (the RaCeR study). Trials. 2019 Jun 6;20(1):328,019-3407-3. 
88. Swindle T, Selig JP, Rutledge JM, Whiteside-Mansell L, Curran G. Fidelity monitoring in complex interventions: A case study of the WISE intervention. Arch Public Health. 2018 Aug 29;76:53,018-0292-2. eCollection 2018.

89. Carroll C, Patterson M, Wood S, Booth A, Rick J, Balain S. A conceptual framework for implementation fidelity. Implement Sci. 2007 Nov 30;2:40,59082-40.

90. Gearing RE, El-Bassel N, Ghesquiere A, Baldwin S, Gillies J, Ngeow E. Major ingredients of fidelity: $A$ review and scientific guide to improving quality of intervention research implementation. Clin Psychol Rev. 2011 Feb;31(1):79-88.

91. Bellg AJ, Borrelli B, Resnick B, Hecht J, Minicucci DS, Ory M, et al. Enhancing treatment fidelity in health behavior change studies: Best practices and recommendations from the NIH behavior change consortium. Health Psychol. 2004 Sep;23(5):443-51.

92. Borrelli B. The assessment, monitoring, and enhancement of treatment fidelity in public health clinical trials. J Public Health Dent. 2011 Winter;71(s1):S52-63.

93. Hoffmann TC, Glasziou PP, Boutron I, Milne R, Perera R, Moher D, et al. Better reporting of interventions: Template for intervention description and replication (TIDieR) checklist and guide. BMJ. 2014 Mar 7;348:g1687.

94. Karas S, Plankis L. Consideration of treatment fidelity to improve manual therapy research. J Man Manip Ther. 2016 Sep;24(4):233-7.

95. O'Shea O, McCormick R, Bradley J, O'Neill B. Fidelity review: A scoping review of the methods used to evaluate treatment fidelity in behavioural change interventions. Physical Therapy Reviews. 2016;21(3-6):207-14.

96. Toomey E, Hardeman W. Addressing intervention fidelity within physical therapy research and clinical practice. J Orthop Sports Phys Ther. 2017 Dec;47(12):895-8.

97. Toomey E, Matthews J, Guerin S, Hurley DA. Development of a feasible implementation fidelity protocol within a complex physical therapy-led selfmanagement intervention. Phys Ther. 2016 Aug;96(8):1287-98. 
98. Boonstra AM, Schiphorst Preuper HR, Reneman MF, Posthumus JB, Stewart RE. Reliability and validity of the visual analogue scale for disability in patients with chronic musculoskeletal pain. Int J Rehabil Res. 2008 Jun;31(2):165-9.

99 Devlin NJ, Krabbe PF. The development of new research methods for the valuation of EQ-5D-5L. Eur J Health Econ. 2013 Jul;14 Suppl 1:S1-3.

100. Devlin NJ, Parkin D, Browne J. Patient-reported outcome measures in the NHS: New methods for analysing and reporting EQ-5D data. Health Econ. 2010 Aug; 19(8): 886-905.

101. SooHoo NF, Samimi DB, Vyas RM, Botzler T. Evaluation of the validity of the foot function index in measuring outcomes in patients with foot and ankle disorders. Foot Ankle Int. 2006 Jan;27(1):38-42.

102. Martinez BR, Staboli IM, Kamonseki DH, Budiman-Mak E, Yi LC. Validity and reliability of the foot function index (FFI) questionnaire brazilian-portuguese version. Springerplus. 2016 Oct 18;5(1):1810,016-3507-4. eCollection 2016.

103. Budiman-Mak E, Conrad K, Stuck R, Matters M. Theoretical model and rasch analysis to develop a revised foot function index. Foot Ankle Int. 2006 Jul;27(7):519-27.

104. Martin RL, Davenport TE, Reischl SF, McPoil TG, Matheson JW, Wukich DK, et al. Heel pain-plantar fasciitis: Revision 2014. J Orthop Sports Phys Ther. 2014 Nov;44(11):A1-33.

105. Budiman-Mak E, Conrad KJ, Mazza J, Stuck RM. A review of the foot function index and the foot function index - revised. J Foot Ankle Res. 2013 Feb $1 ; 6(1): 5,1146-6-5$.

106. Landorf KB, Radford JA. Minimal important difference: Values for the foot health status questionnaire, foot function index and visual analogue scale. The Foot. 2008 March 2008;18(1):15-9.

107. Walmsley S, Williams $A E$, Ravey $M$, Graham $A$. The rheumatoid foot: $A$ systematic literature review of patient-reported outcome measures. J Foot Ankle Res. 2010 Jul 9;3:12,1146-3-12. 
108. Martin RL, Irrgang J]. A survey of self-reported outcome instruments for the foot and ankle. J Orthop Sports Phys Ther. 2007 Feb;37(2):72-84.

109. McPoil TG, Martin RL, Cornwall MW, Wukich DK, Irrgang JJ, Godges J]. Heel pain--plantar fasciitis: Clinical practice guildelines linked to the international classification of function, disability, and health from the orthopaedic section of the american physical therapy association. J Orthop Sports Phys Ther. 2008 Apr;38(4):A1-A18.

110. Martin RL, Irrgang J], Burdett RG, Conti SF, Van Swearingen JM. Evidence of validity for the foot and ankle ability measure (FAAM). Foot Ankle Int. 2005 Nov;26(11):968-83.

111. Ritchie J, Lewis J, McNaughton Nicholls C, Ormston R. Qualitative research practice: A guide for social science students and researchers. Second ed. Los Angeles: Sage; 2014.

112. Novick G. Is there a bias against telephone interviews in qualitative research? Res Nurs Health. 2008 Aug;31(4):391-8.

113. Drabble L, Trocki KF, Salcedo B, Walker PC, Korcha RA. Conducting qualitative interviews by telephone: Lessons learned from a study of alcohol use among sexual minority and heterosexual women. Qual Soc Work. 2016 Jan;15(1):118-33.

114. Green J, Thorogood N. Qualitative methods for health research. 4th ed. Los Angeles: Sage; 2018.

115. Munro A, Bloor M. Process evaluation: The new miracle ingredient in public health research? Qualitative Research. 2010 12/01; 2020/05;10(6):699-713.

116. Doyle C, Lennox L, Bell D. A systematic review of evidence on the links between patient experience and clinical safety and effectiveness. BMJ Open. 2013 Jan 3;3(1):10.1136/bmjopen,2012-001570.

117. Poltawski L, Norris M, Dean S. Intervention fidelity: Developing an experience-based model for rehabilitation research. J Rehabil Med. 2014 Jul;46(7):609-15. 
118. Brinkmann S, Kvale S. Doing interviews. Los Angeles: Sage; 2018; 2018.

119. Miller FG. The ethical challenges of human research: Selected essays. New York: Oxford University Press; 2012; 2012.

120. Creswell JW, Creswell JD. Research design: Qualitative, quantitative and mixed methods approaches. 5th; International student ed. Los Angeles: Sage; $2018 ; 2018$.

121. Hicks C. Research methods for clinical therapists: Applied project design and analysis. 5th ed. Edinburgh: Churchill Livingstone/Elsevier; 2009.

122. Resnik DB. The ethics of research with human subjects: Protecting people, advancing science, promoting trust. Cham, Switzerland: Springer; 2018.

123. Polgar S, Thomas SA. Introduction to research in the health sciences. Sixth ed. Edinburgh: Churchill Livingstone; 2013.

124. Pierce J, Jameton A. The ethics of environmentally responsible health care. Oxford: Oxford University Press; 2004. 